\title{
BMJ Open Exploring family based immigrant youth substance use prevention programmes: a scoping review protocol
}

\author{
Yiyan Li (D) , ${ }^{1}$ Geoffrey Maina (D) , ${ }^{1}$ Mamata Pandey, ${ }^{2}$ Jonathan Amoyaw, ${ }^{3}$ \\ Yiting Fang ${ }^{1}$
}

To cite: Li Y, Maina G, Pandey $\mathrm{M}$, et al. Exploring family based immigrant youth substance use prevention programmes: a scoping review protocol. BMJ Open 2021;11:e046766. doi:10.1136/ bmjopen-2020-046766

- Prepublication history and additional supplemental material for this paper are available online. To view these files, please visit the journal online (http://dx.doi.org/10.1136/ bmjopen-2020-046766).

Received 09 November 2020 Accepted 17 May 2021

\section{Check for updates}

(c) Author(s) (or their employer(s)) 2021. Re-use permitted under CC BY-NC. No commercial re-use. See rights and permissions. Published by BMJ.

${ }^{1}$ College of Nursing, University of Saskatchewan, Prince Albert, Saskatchewan, Canada

${ }^{2}$ Saskatchewan Health Authority, Regina, Saskatchewan, Canada ${ }^{3}$ Department of Sociology and Social Anthropology, Dalhousie University, Halifax, Nova Scotia, Canada

Correspondence to

Dr Yiyan Li; yiyan.li@usask.ca

\section{ABSTRACT}

Introduction Canada has one of the highest rates of problematic opiate and alcohol use in the world. Globally, Canada was the second country that legalized marijuana for non-medical use. As Canada is an immigrant-receiving country, newcomers and immigrants contend with a substance use landscape that was likely absent in their countries of origin. Although immigrants have lower rates of substance use than the host population, the risk of substance use, especially among youth, increases with acculturation and peer pressure. While parents are best placed to mitigate the risks for substance use among their youth, immigrant parents often do not have the knowledge and skills to do so. Therefore, culturally adaptable family based interventions need be explored to build immigrant parents' capacities to mitigate substance use risks. Aim and purpose The aim of this scoping review is to explore family based substance use prevention interventions for immigrant youth, which will be guided by two questions:

1. What is known about family based interventions for preventing immigrant adolescents' substance use?

2. What are the features and study results of these intervention protocols?

Methods and analysis We will apply Arksey and 0'Malley's procedure for reporting scoping review and report study findings based on the Preferred Reporting Items for Systematic Reviews and Meta-Analyses guidelines for scoping reviews.

Discussion We hope that the knowledge translation emanating from this review will increase immigrant parents' knowledge of substance use and enable them to effectively intervene to prevent substance use among their youth. We also hope that this work can inform policy development on best practices for substance use prevention and for the creation of culturally sensitive programmes and services for immigrant youth.

\section{BACKGROUND}

\section{Substance and addiction landscape in Canada}

The use of both licit and illicit substances is a growing problem in Canada. ${ }^{1}$ Canada has the second-highest consumption of prescription opioids in the world due to overprescription and non-medical use of these strong analgesics. ${ }^{1-3}$ The use of opioids for non-medical purposes for both licit and illicit drugs is a

\section{Strengths and limitations of this study}

This study will provide insights into family focused substance use prevention interventions for immigrant youth that can be adapted and applied to a local setting.

- This study will help reorient the role of families in substance use prevention and identify strategies that may help families play that role.

- The scoping review is limited to family based, immigrant-focused substance use interventions that are published in English.

- The exclusion of interventions that do not target immigrants may limit the research by excluding relevant programmes that may be applicable to the population of interest

serious public health problem causing opioid use disorder, a problematic pattern of opioid use that leads to clinically significant issues, and significantly impacts people's qualities of life, occupations and relationships. ${ }^{2-4}$ In Canada, more and more youth has the issue of opioid overdoses, which requires hospitalization. ${ }^{5}$ Alcohol use among children in grades $7-12$ is high, with $44 \%$ reporting alcohol consumption in 2018. ${ }^{6}$ In Ontario, about $14 \%$ of teens have reported using prescription opioids for extra-medical use. ${ }^{7}$ In Canada, about $55 \%$ of vehicle accidents that involve youth are caused by alcohol or drugs, and car accidents are the main factor which causes youth death. ${ }^{8}$

In 2018, Canada became the second country to legalise cannabis, ${ }^{9}$ which is the second-most used substance in Canada. ${ }^{10}$ In the third quarter of 2019, about $26 \%$ of adolescents and young adults (aged 15-24 years) reported having used cannabis, ${ }^{11}$ while $18 \%$ of students (about 374,000) in grades 7-12 reported using cannabis in 2018-2019. ${ }^{6}$ Adolescents' cannabis use is associated with an increased risk of mental health illnesses, poor academic outcomes and personal 
disadvantages. ${ }^{12}$ Methamphetamine use is also prevalent among youth and young adults, with $1.2 \%$ of Canadian students in grades 7-12 reporting using it in $2015 .{ }^{13} \mathrm{In}$ 2017-2018, 23580 hospitalisations among youth were caused by substance misuse. ${ }^{14}$ Among these youth, about $17 \%$ of them had more than one hospitalisation in the same year and about two-thirds of them had concurrent mental health issues. ${ }^{14}$

\section{Immigrants and attitude towards substance use}

Canada is a popular destination for international migration, and immigrants have become an important driver of population and economic growth. The 2016 Census showed that immigrants accounted for $21.9 \%$ of the Canadian population. ${ }^{15}$ In 2017-2018, $80 \%$ of Canada's population growth was driven by immigrants, and in 2019, 313580 new immigrants landed in Canada. ${ }^{16} 17$ Although Canada pursues a multicultural policy, immigrants to Canada, especially the youth acculture at a higher rate than adults. ${ }^{18} 19$ The prevailing sociocultural climate of the destination country, that is, Canada, impacts how immigrants and newcomers acculturate. ${ }^{20}$ One of the realities that immigrants are confronted with is the perversity of substance use that differs from their countries of origin. ${ }^{21}$ Yet, although new immigrant youth are less likely to experiment with substances, their risk of substance use increases with their length of stay. ${ }^{22}$ In so doing, they embrace the host nations' culture and behaviors that their parents may deem risky or unsafe. ${ }^{20} 23$

Immigrants' attitudes towards substances may be informed by availability and acceptability of these substances at home and in their host country. For instance, immigrants who come from countries where plant products are commonly used are more likely to accept cannabis than those who do not. ${ }^{24}{ }^{25}$ Also, since alcohol and marijuana are easily accessible and socially acceptable substances in Canada, immigrants without prior exposure to them may be inclined to start using them for recreation purposes. ${ }^{26}$ Moreover, immigrants' risk for substance use might be aggravated by immigration-related stress, which increases the risk of developing mental health illnesses. ${ }^{27}$ Thus, new immigrants may use substances as a maladaptive coping mechanism to deal with stress induced by immigration and resettlement processes.

\section{Immigrant youth and risk of substance use}

Generally, new immigrant youth in Canada have a significantly lower level of substance use than their Canadian counterparts. For instance, a study by Hamilton $e t$ al on subjective social status, immigration generations and substance use among youth, ${ }^{28} 13.9 \%$ of first-generation immigrant youth in Ontario reported using cannabis before, while $9.8 \%$ drink alcohol regularly. In the same research, $22.6 \%$ of second-generation immigrant adolescents reported having used cannabis, and $16.8 \%$ consumed alcohol regularly. ${ }^{28}$ The acculturation process has been cited as a risk factor for the initiation of substance use among immigrant youth. ${ }^{24} 29$
Thus, immigrant youth may feel pressured to conform to the practices of their receiving country and feel the need to belong with their peers who may be using substances. $^{24} 2930$

To mitigate the risks of substance use for immigrants, family based substance use prevention programmes are needed. Such programmes must be culturally safe and empowering to parents with effective strategies to help youth navigate these risks. ${ }^{32}{ }^{32}$ Cultural safety is underpinned by principles of social justice and is grounded in critical theoretical perspectives, and draws attention to the impact of racialisation, culturalisation, discrimination and disparities in health and access to healthcare among marginalised communities. ${ }^{33} 34$ Family based substance use prevention programmes can be implemented in group settings with active family participation through role-plays, curriculum-based approaches, videos and youth-parent collaborative activities. ${ }^{33-36}$

Due to the increased risk of substance use among immigrant youth in Canada, the research team, majority of whom are first-generation immigrants with diverse lived experiences, together with stakeholders involved in settlement programmes identified a need to equip parents, guardians and caregivers with skills to mitigate this risk. This scoping review is guided by principles of communitybased research, and as such, the research focus was informed by community partners. Any intervention that will emanate from this review will also be selected by the community partners. Community consultation is essential in grounding this review to foster ownership of the process and the outcome. ${ }^{37}$ Therefore, this consultation yielded the following objectives that guide the scoping review: (a) to understand substance use trends and the risks that their kids may encounter; (b) to detect early signs of substance use among youth and (c) identify resources to mitigate the risks for immigrant youth substance use and to build capacity for families to intervene to delay or prevent substance use initiation. Although the definition of youth varies, for the purpose of this review, youth will be referred to those who range between 12 years and 17 years of age.

Studies suggest that parents are important resources for substance use prevention among immigrant youth. Effective parenting, characterised by parental warmth and close supervision of children, can delay or prevent substance use initiation. ${ }^{30}{ }^{38}$ In addition, parents' zero tolerance of and clear rules about substance use can prevent substance use initiation. ${ }^{38} 4041$ A good family based substance use prevention programme for immigrant youth need to consider skills and behavioural and cultural factors when developing or adapting it. ${ }^{37}$ These include understanding language limitations, the acculturation process and the available support systems. It also needs to highlight family's role in protecting and supporting youth in the acculturation process. ${ }^{42}$ 


\section{AIM AND PURPOSE}

The aim of this review is to explore the types and characteristics of family based substance use prevention interventions for immigrant youth. The purpose of this project is to identify and describe family based substance use prevention interventions that could potentially be adapted to support immigrant families in mitigating the risk of substance use initiation facing youth.

\section{METHODS}

We will conduct a scoping review of family based substance use prevention programmes for immigrant youth to identify and describe these interventions. A scoping literature review focuses on an under-researched or complicated research area and seeks to summarise key concepts, principles, primary sources and evidence under that topic. ${ }^{43}$ This scoping review will be guided by community-based research principles, a collaborative, equal partnership between researchers and community members during the research process. ${ }^{37445}$ We will depend on stakeholders within the immigrant community to identify and refine the research problem, develop an appropriate knowledge translation tool and thereafter seek mechanisms for developing a culturally appropriate intervention for immigrant families. ${ }^{45}$ This scoping review started with the consultation with community partners involved in providing settlement and integration services for immigrants. These partners will later be constituted to a community advisory committee (CAC) and will oversee the completion of the scoping review, knowledge translation and development of an intervention suitable for the targeted community. Immigrant youth will be involved in the knowledge event where the research team will present the results. Also, those youth will provide feedback regarding the review's findings. The scoping review will be guided by Arksey and O'Malley's ${ }^{46}$ steps of conducting a scoping review, which are outlined below.

\section{Step 1: identify the research question}

Through community consultation, the search terms were suggested, which contributed to the formulation of research questions. A search has been conducted on PubMed, Web of Science and PROSPERO to ensure that no such scoping review has been done. The scoping review will be guided by two broad research questions: (a) 'what is known about family based interventions for preventing immigrant youth's substance use?' and (b) 'what are the features and study results of these intervention protocols?'.

\section{Step 2: identify relevant studies}

The research team, with the help of a health sciences librarian, has developed a strategy to search for published articles and the grey literature. A concept map based on the following keywords has been developed to guide the search for relevant publications: 'family', 'prevention', 'immigration', 'youth', 'substance use' and types of substances. Online supplemental appendix 1 is a prototype of a concept map that will be used to guide the review. A test search on MEDLINE has been conducted to assess its feasibility. Thereafter, the following databases will be searched for relevant studies: Ovid MEDLINE, 1946-present; CINAHL (EBSCO); EMBASE, 1947present; Web of Science, 1900-present; and APA PsycInfo, 1806-present. This scoping review will include literature published between 2000 and 2020. Limiting publications in the past 20 years is because substance use dynamics and interventions are changing, and interventions implemented more than 20 years ago may not suitable for the current situation. ${ }^{47}$ All relevant studies will be downloaded and saved in Mendeley, a referencemanaging software, and all duplicate references will be removed. Besides searching literature in databases, the grey literature will also be included in this scoping review. The research team will search for the grey literature from Canadian organisations related to substance use, including the Canadian Centre on Substance Use and Addiction (CCSA), the Centre for Addiction and Mental Health (CAMH) and the Canadian Agency for Drugs and Technologies in Health (CADTH). Searching for the grey literature will be based on the keywords in the concept map, and specific steps will be adjusted based on the search engines' results.

Both the CCSA and CAMH are leading Canadian research centres for substance use and addiction, providing guidelines to care, research and education. ${ }^{48} 49$ The CADTH is a non-profit organisation that provides evidence for healthcare decision-makers. ${ }^{50}$ The keywords used to search the electronic databases will be applied for the grey literature search.

\section{Inclusion criteria}

To be included in the scoping review, studies must (a) discuss a family focused substance use prevention intervention; (b) empirical studies except reviews; (c) have the immigrant youth (aged 12-17 years) as the intervention target; (d) be published in English and (e) be conducted in Canada, the USA, Europe, Australia or New Zealand, as these regions are key destinations for international migrants. Also, considering the development stage and needs to be supervised by parents, participants' age in this study will be between 12 years and 17 years.

\section{Exclusion criteria}

Interventions that are not family focused, focus on youth older than 17 years or focus on native-born populations will not be included in the study. Also, literature reviews, letters to the editors and opinion pieces will be excluded from the study.

\section{Step 3: study selection}

The search results will be exported to a reference management system, Rayyan, and the inclusion and exclusion criteria will be applied. The research team will screen for title/abstract first to determine the suitability of the 
article to be included in the review. Two reviewers will be involved in the screening process. A third reviewer will be involved as a tie breaker in instances where the two reviewers cannot agree on whether to include or exclude an article.

\section{Step 4: charting the data}

The research team will extract information from the articles using a table with the following columns: (a) name(s) of author(s), year of publication and title, (b) characteristics of intervention and (c) outcomes and significant findings.

\section{Step 5: collating, summarising and reporting results}

The goal of conducting the scoping review is to map out findings and provide an overview of selected literature. ${ }^{46}$ We will report the research results based on the Preferred Reporting Items for Systematic Reviews and MetaAnalyses guidelines, a method for reviewing literature that is useful in reviewing healthcare interventions. ${ }^{51} 52$ Therefore, first, we will provide a basic numerical description of the articles included in the study, including their locations, the interventions studied and the approaches used in the interventions. Second, we will synthesis the data and identify common themes that emanate from the extracted data. A narrative description of these themes will be developed to convey the main findings.

\section{Step 6: sharing the study findings/patient and public involvement}

We will present this scoping review's preliminary findings to the CAC and thereafter to the wider stakeholders involved in providing services to newcomers and immigrants with limited English abilities. During this meeting, we will seek their feedback regarding the presentation, scoping review's findings and initiate the process of identifying an intervention from the presentation that can be adapted to serve the local immigrant community. Data collected in this phase will inform grant applications for intervention development and implementation.

\section{DISCUSSION}

The decision to conduct a scoping review of family based substance use prevention interventions for immigrant youth has been informed by stakeholders, who observed that there is a dearth of literature on substance use interventions and programming that focus on immigrant youth. Moreover, immigrant parents have expressed a desire to build capacity to prevent and mitigate the risks of substance use that the youth face. Parents' active engagement in preventing their youth's substance use can help youth realise the consequences of substance use and hopefully help them avoid engaging in these practices. ${ }^{3840}$ The scoping review is, therefore, set in such a manner that it applies the community-based research principles from the conceptualisation of the idea to the eventual implementation of a family-based substance use prevention intervention for immigrant youth. Researchers and community stakeholders are set to work collaboratively in hopes that this partnership will increase parental knowledge of substance use, address critical societal issues related to substance use, contribute to public health and enhance ownership and uptake of the research findings. ${ }^{53} 54$

This research project is grounded on the understanding that newcomers and immigrants are a vulnerable population. While they may have better health indicators than the native population at the time of immigration, this advantage tends to wane with the increasing length of stay in the host country. ${ }^{55}$ Therefore, supporting newcomers and immigrants navigate one of the imminent risks that they face even before they acclimatise and settle in their new country is needed. This work also takes a social justice lens because of the socioeconomic and health inequalities that newcomers and immigrants face, which make them vulnerable to health conditions. For instance, besides contending with cultural shock, racial issues and a new way of life, most newcomers and immigrants often struggle to secure employment that matches their skills and qualifications. ${ }^{56}$ Hence, most are at a higher risk of living in poverty than the native population. ${ }^{57}$ This low socioeconomic status increases their risks of developing mental illnesses, limits their ability to parent effectively and even increases their risk of engaging in maladaptive behaviours to deal with the ensuing stress. ${ }^{2758}$

Immigrant parents are important resources for the youth and can influence their behaviour by transmitting values, beliefs and rules on substance use. ${ }^{38}$ However, raising children and supporting youth in a foreign country with no social support or extended family may prove to be an additional challenge for many parents who already face cultural and social challenges. ${ }^{59} 60$ Nevertheless, resources for immigrants that would help them avoid substance use are urgently required. Early onset of substance use increases risks for poor educational outcomes, substance use disorders, substance-related death and even suicides. ${ }^{61}{ }^{62}$ Such resources could help immigrant youth avoid behaviours and practices that would hinder their ability to achieve academic and future success. $^{6364}$

This scoping review could contribute to the growing knowledge about immigrants' substance use, inform policy development on best practices in substance use prevention programmes for immigrant youth and help create culturally sensitive programmes and services for the immigrant population.

\section{LIMITATIONS OF THE REVIEW}

Limiting the studies included in this review to those published in the English language means that the literature that would have been relevant for our study will be omitted. Also, including only substance use prevention interventions aimed at immigrant youth deprives this 
study of potentially relevant interventions that could be culturally adapted to support the affected community.

\section{PATIENT AND PUBLIC INVOLVEMENT}

Since this review is guided by the principles of communitybased research, CAC will be actively involved in all the phases of the study, including knowledge translation and dissemination and selection of an intervention. The research team will share the findings of the study with the targeted community in the region by organising a 1-day knowledge exchange event. Thereafter, the community will provide feedback on the content and initiate the process of identifying an intervention that can be adapted to address community needs.

Acknowledgements We thank Kevin Read (Librarian, Leslie and Irene Dubé Health Sciences Library, University of Saskatchewan) for his insightful suggestions on developing search strategies.

Contributors YL was responsible for writing the protocol. GM provided in-depth input to the protocol by refining the protocol and providing edits and suggestions. He proofread the drafts and approved the final manuscript. MP and JA edited and proofread the protocol. YF reviewed the final draft and provided feedback.

Funding This scoping review has been supported by Canadian Research Initiative in Substance Misuse.

Competing interests None declared.

Patient consent for publication Not required.

Provenance and peer review Not commissioned; externally peer reviewed.

Supplemental material This content has been supplied by the author(s). It has not been vetted by BMJ Publishing Group Limited (BMJ) and may not have been peer-reviewed. Any opinions or recommendations discussed are solely those of the author(s) and are not endorsed by BMJ. BMJ disclaims all liability and responsibility arising from any reliance placed on the content. Where the content includes any translated material, BMJ does not warrant the accuracy and reliability of the translations (including but not limited to local regulations, clinical guidelines, terminology, drug names and drug dosages), and is not responsible for any error and/or omissions arising from translation and adaptation or otherwise.

Open access This is an open access article distributed in accordance with the Creative Commons Attribution Non Commercial (CC BY-NC 4.0) license, which permits others to distribute, remix, adapt, build upon this work non-commercially, and license their derivative works on different terms, provided the original work is properly cited, appropriate credit is given, any changes made indicated, and the use is non-commercial. See: http://creativecommons.org/licenses/by-nc/4.0/.

\section{ORCID iDs}

Yiyan Li http://orcid.org/0000-0001-6372-7600

Geoffrey Maina http://orcid.org/0000-0003-4982-9656

\section{REFERENCES}

1 Belzak L, Halverson J. The opioid crisis in Canada: a national perspective. Health Promot Chronic Dis Prev Can 2018;38:224-33.

2 Degenhardt L, Grebely J, Stone J, et al. Global patterns of opioid use and dependence: harms to populations, interventions, and future action. Lancet 2019;394:1560-79.

3 Strang J, Volkow ND, Degenhardt L, et al. Opioid use disorder. Nat Rev Dis Primers 2020:6:3.

4 American Psychiatric Association. Diagnostic and statistical manual of mental disorders (DSM-5). American Psychiatric Association, 2013.

5 Government of Canada. Canada's Opioid Crisis (fact sheet). Gov. Canada, 2019. Available: https://www.canada.ca/en/health-canada/ services/publications/healthy-living/canada-opioid-crisis-fact-sheet. html

6 Government of Canada. Summary of results for the Canadian student tobacco, alcohol and drugs survey 2018-19, 2019. Available: https://www.canada.ca/en/health-canada/services/canadianstudent-tobacco-alcohol-drugs-survey/2018-2019-summary.html

7 The Centre for Addiction and Mental Health (camh). Youth and prescription painkillers. cent. addict. MENT. heal. Available: https:// www.camh.ca/en/health-info/guides-and-publications/youth-andprescription-painkillers

8 Drug Free Kids. Youth and alcohol, 2021.

9 Canadian Centre on Substance Use and Addiction (CCSA). Cannabis (Canadian drug summary), 2020. Available: https://www.ccsa. $\mathrm{ca} /$ sites/default/files/2020-08/CCSA-Canadian-Drug-SummaryCannabis-2020-en.pdf

10 Ferreras J. Marijuana is legal everywhere across Canada now. Glob. news, 2018. Available: https://globalnews.ca/news/4559085/ marijuana-legalization-canada-midnight/

11 Statistics Canada. National cannabis survey, third quarter 2019, 2020Statistics Canada. Available: https://www150.statcan.gc.ca/n1/ daily-quotidien/191030/dq191030a-eng.htm

12 Government of Canada. Strengthening Canada's Approach to Substance Use Issues. Gov. Canada, 2018.

13 Canadian Centre on Substance Use and Addiciton (CCSA) Methamphetamine, 2020Canadian Centre on Substance Use and Addiciton (CCSA). Available: https://www.ccsa.ca/sites/default/files/ 2020-03/CCSA-Canadian-Drug-Summary-Methamphetamine-2020en.pdf

14 Canadian Institute for Health Information. Hospital stays for harm caused by substance use among youth age 10 to 24, 2019: 1-22.

15 Statistics Canada. Focus on geography series, 2016 census. STAT. Canada, 2019. Available: https://www12.statcan.gc.ca/censusrecensement/2016/as-sa/fogs-spg/Facts-CAN-Eng.cfm?TOPIC=2\& LANG=Eng\&GK=CAN\&GC $=01$

16 Government of Canada. Annual report to Parliament on immigration, 2018: 1-43. https://www.canada.ca/content/dam/ircc/migration/ircc/ english/pdf/pub/annual-report-2018.pdf

17 Statistics Canada. Canada's population estimates: Age and sex, July 1, 2019, 2019. Available: https://www150.statcan.gc.ca/n1/en/dailyquotidien/190930/dq190930a-eng.pdf?st=nU9UcapR

18 Berry JW, Hou F. Immigrant acculturation and wellbeing in Canada. Can Psychol 2016;57:254-64.

19 Schwartz SJ, Unger JB, Zamboanga BL. Rethinking the concept of Acculturation. Am Psychol 2010;65:237-51.

20 Bui HN. Racial and ethnic differences in the immigrant paradox in substance use. J Immigr Minor Health 2013;15:866-81.

21 Charles CZ. Won't you be my neighbor: Race, class, and residence in Los Angeles. Russell Sage Foundation, 2006.

22 Rumbaut R. Children of immigrants and their achievement: the roles of family, Acculturation, social class, gender, ethnicity, and school context. Addressing Achiev gap Theory informing Pract, 2005: 1-22.

23 Gordon MM. Assimilation in American life: the role of race, religion, and national origins. Oxford University Press on Demand, 1964.

24 McCann M. Drug knowledge, attitudes, beliefs and use among Chinese international students on the West coast of the US and Canada. Saint Mary's College of California, 2018.

25 Moloney M, Hunt G, Evans K. Asian American identity and drug consumption: from acculturation to normalization. J Ethn Subst Abuse 2008;7:376-403.

26 Borges G, Medina-Mora ME, Breslau J, et al. The effect of migration to the United States on substance use disorders among returned Mexican migrants and families of migrants. Am J Public Health 2007:97:1847-51.

27 Robert AM, Gilkinson T. Mental health and well-being of recent immigrants in Canada: evidence from the longitudinal survey of immigrants to Canada. Citizsh Immigr Canada, 2012: 1-33. https:// www.canada.ca/content/dam/ircc/migration/ircc/english/pdf/ research-stats/mental-health.pdf\%0Ahttp://site.ebrary.com.ezproxy. library.dal.ca/lib/dal/docDetail.action?doclD=10726283

28 Hamilton HA, van der Maas M, Boak A, et al. Subjective social status, immigrant generation, and cannabis and alcohol use among adolescents. J Youth Adolesc 2014;43:1163-75.

29 Burlew AK, Johnson CS, Flowers AM, et al. Neighborhood risk, parental supervision and the onset of substance use among African American adolescents. J Child Fam Stud 2009;18:680-9.

30 Myers R, Chou C-P, Sussman S, et al. Acculturation and substance use: social influence as a mediator among Hispanic alternative high school youth. J Health Soc Behav 2009;50:164-79.

31 Williams LR, Ayers S, Baldwin A, et al. Delaying youth substance-use initiation: a cluster randomized controlled trial of complementary youth and parenting interventions. J Soc Social Work Res 2016:7:177-200.

32 Delforterie MJ, Verweij KJH, Creemers HE, et al. Parental solicitation, parental control, child disclosure, and substance use: native and immigrant Dutch adolescents. Ethn Health 2016;21:535-50. 
33 Kirkham SR, Browne AJ. Toward a critical theoretical interpretation of social justice discourses in nursing. Advances in Nursing Science 2006;29:324-39.

34 Curtis $\mathrm{E}$, Jones $\mathrm{R}$, Tipene-Leach $\mathrm{D}$, et al. Why cultural safety rather than cultural competency is required to achieve health equity: a literature review and recommended definition. Int J Equity Health 2019;18:1-17.

35 Daley DC. Family and social aspects of substance use disorders and treatment. J Food Drug Anal 2013;21:S73-6.

36 American Dietetic Association (ADA). Position of the American dietetic association: individual-, family-, school-, and communitybased interventions for pediatric overweight. J Am Diet Assoc 2006;106:925-45.

37 Adams N. Using community engagement to map pathways of opioid use and recovery. J Community Health Nurs 2020;37:1-8.

38 Özdemir M, Koutakis N. Does promoting parents' negative attitudes to underage drinking reduce adolescents' drinking? the mediating process and moderators of the effects of the Örebro prevention programme. Addiction 2016;111:263-71.

39 Trucco EM, Colder CR, Wieczorek WF. Vulnerability to peer influence: a moderated mediation study of early adolescent alcohol use initiation. Addict Behav 2011;36:729-36.

40 Koning IM, van den Eijnden RJJM, Engels RCME, et al. Why target early adolescents and parents in alcohol prevention? the mediating effects of self-control, rules and attitudes about alcohol use. Addiction 2011;106:538-46.

41 Villagrana M, Lee S-Y. Parental influence on adolescent cigarette, alcohol and marijuana use: a focus on Race/Ethnicity and age. Child Adolesc Soc Work J 2018;35:107-18.

42 Slobodin O, de Jong JTVM. Family interventions in traumatized immigrants and refugees: a systematic review. Transcult Psychiatry 2015:52:723-42.

43 Mays N, Roberts E, Popay J. Synthesising research evidence. In: Fulop N, Allen P, Clarke A, et al, eds. Studying the organization and delivery of health services: research methods. London: Routledge, 2001: 188-220.

44 Simonds VW, Wallerstein N, Duran B, et al. Community-Based participatory research: its role in future cancer research and public health practice. Prev Chronic Dis : 2013;10:E78.

45 Strand K, Marullo S, Cutforth N. Principles of best practice for community-based research. Michigan J Community Serv Learn 2003;9:5-15

46 Arksey H, O'Malley L. Scoping studies: towards a methodological framework. Int J Soc Res Methodol 2005;8:19-32.

47 Maina G, Phaneuf T, Kennedy M, et al. School-Based interventions for preventing substance use in Indigenous children ages 7-13: a scoping review protocol. BMJ Open 2020;10:e034032-6.

48 Canadian Centre on Substance Use and Addiction (CCSA). Our focus. can. cent. Subst. Use addict, 2021.
49 The Centre for Addiction and Mental Health (camh). About CAMH. cent. addict. MENT. heal. Available: https://www.camh.ca/en/drivingchange/about-camh

50 Canadian Agency for Drugs and Technologies in Health (CADTH). About CADTH. can. agency drugs Technol. heal, 2021. Available: https://www.cadth.ca/about-cadth

51 Moher D, Liberati A, Tetzlaff J, et al. Preferred reporting items for systematic reviews and meta-analyses: the PRISMA statement. PLoS Med 2009;6:e1000097.

52 Tricco AC, Lillie E, Zarin W, et al. PRISMA extension for scoping reviews (PRISMA-ScR): checklist and explanation. Ann Intern Med 2018;169:467-73.

53 Stanton TK. New times demand new scholarship: opportunities and challenges for civic engagement at research universities. Educ Citizsh Soc Justice 2008;3:19-42.

54 Wallerstein NB, Duran B. Using community-based participatory research to address health disparities. Health Promot Pract 2006;7:312-23.

55 Vang ZM, Sigouin J, Flenon A, et al. Are immigrants healthier than native-born Canadians? A systematic review of the healthy immigrant effect in Canada. Ethn Health 2017;22:209-41.

56 Hudon T. Women in Canada : A Gender-based Statistical Report Immigrant Women. 7th Edn. Women in Canada, 2015: 1-39.

57 Chui T. Women in Canada: a gender-based statistical report. Statistics Canada, 2011

58 Beiser M, Hou F, Hyman I, et al. Poverty, family process, and the mental health of immigrant children in Canada. Am J Public Health 2002;92:220-7.

59 Singh S, McBride K, Kak V. Role of social support in examining Acculturative stress and psychological distress among Asian American immigrants and three sub-groups: results from NLAAS. $J$ Immigr Minor Health 2015;17:1597-606.

60 BORNSTEIN M, BOHR Y. Immigration, Acculturation and parenting. Encycl Early Child Dev, 2011: 1-8. http://www.child-encyclopedia. com/documents/Bornstein-BohrANGxp1.pdf

61 Mundt MP. The impact of peer social networks on adolescent alcohol use initiation. Acad Pediatr 2011;11:414-21.

62 Wen X, Shenassa ED. Interaction between parenting and neighborhood quality on the risk of adolescent regular smoking Nicotine Tob Res 2012;14:313-22.

63 Enriquez L. "Because We Feel the Pressure and We Also Feel the Support": Examining the Educational Success of Undocumented Immigrant Latina/o Students. Harv Educ Rev 2011;81:476-500.

64 Jaffe-Walter R, Lee SJ. "To Trust in My Root and to Take That to Go Forward": Supporting College Access for Immigrant Youth in the Global City. Anthropol Educ Q 2011;42:281-96. 